\title{
Vector Tick Transmission Model of Spotted Fever Rickettsiosis
}

\author{
Tais B. Saito, ${ }^{*}$ Jeremy Bechelli, ${ }^{*}$ Claire Smalley, ${ }^{*}$ Shahid Karim, ${ }^{\dagger}$ and David H. Walker*
}

From the Department of Pathology, * University of Texas Medical Branch at Galveston, Galveston, Texas; and the Department of Biological Sciences, ${ }^{\dagger}$ University of Southern Mississippi, Hattiesburg, Mississippi

\author{
Accepted for publication \\ September 10, 2018. \\ Address correspondence to Tais \\ B. Saito, D.V.M., M.S., Ph.D., \\ Department of Pathology, Uni- \\ versity of Texas Medical \\ Branch at Galveston, 301 Uni- \\ versity Blvd., Route 0609, \\ Galveston, TX 77555. E-mail: \\ tbsaito@utmb.edu.
}

\begin{abstract}
Many aspects of rickettsial infections have been characterized, including pathogenic and immune pathways and mechanisms of rickettsial survival within the vertebrate host and tick vector. However, very few studies are focused on the complex pathogen-vector-host interactions during tick feeding. Therefore, our objective was to develop a tick transmission model of the spotted fever group of rickettsial infections to study the initial events in disease development. The most appropriate strain of mouse was identified for evaluation as a transmission model, and the course of infection, bacterial levels, histopathologic changes, and antibody response during tick transmission in mice infested with Amblyomma maculatum ticks carrying the emerging pathogen, Rickettia parkeri, were studied. Results showed distinct clinical signs in $\mathrm{C} 3 \mathrm{H} / \mathrm{HeN}$ mice infected intravenously, leading to selection of this mouse strain for tick transmission studies. Active infection of animals was observed after tick vector transmission. The bacteria disseminated systemically and spread to several organs at 24 hours after tick attachment, with peak bacterial load at day 6 after tick attachment. Skin, lung, and liver showed the greatest pathologic changes, with inflammatory cellular infiltration and necrosis. These findings indicate the feasibility of using murine infection with $R$. parkeri by $A$. maculatum tick transmission as a model to study different aspects of the spotted fever group of rickettsial disease establishment. (Am J Pathol 2019, 189: 115-123; https://doi.org/10.1016/j.ajpath.2018.09.005)
\end{abstract}

Several species of the spotted fever group (SFG) Rickettsia infect animals and humans worldwide. Severe disease has been observed in humans exposed to ticks carrying Rickettsia rickettsii and Rickettsia conorii. Rickettsial pathogens are prevalent in particular geographic regions determined in large part by the vector. In the United States, Rickettsia parkeri causes disease in humans after transmission, primarily via Amblyomma maculatum ticks, but under experimental conditions Amblyomma americanum also can serve as a competent vector. $^{1,2}$

R. parkeri rickettsiosis was first described in 2004 as a human disease. Through June 2016, there have been 39 cases, predominantly from the southeastern United States, documented in the scientific literature or confirmed by laboratory assays at the CDC. ${ }^{3,4}$ However, the true incidence of $R$. parkeri rickettsiosis in the United States is unknown. Serologic assays currently used to diagnose SFG rickettsial infections lack species-specificity because there is considerable crossreactivity among pathogens. It is likely that some of the approximately 13,500 noncharacterized cases of SFG rickettsioses reported in the United States during 2008 to 2012 were caused by $R$. parkeri. $^{5}$

$R$. parkeri has been incriminated as the most important pathogen of spotted fever rickettsiosis in Uruguay, Argentina, and parts of Brazil. ${ }^{6-9}$ With increasing incidence of these infections, it is important to understand the potential for disease development and to study the immune responses to SFG rickettsiae. Many animal models have been developed but are incomplete because of limitations in the potential approaches to the host immune system, or they lack the aspect of tick transmission in understanding the pathogenesis of these diseases. Needle-injected murine models of SFG rickettsial

Supported by The Carmage and Martha Walls Distinguished University Chair in Tropical Diseases.

Disclosures: None declared.

Current address of J.B., Department of Biological Sciences, Sam Houston State University, Huntsville, TX. 
infections have been developed that mimic the various human pathologies observed during mild and severe forms of the diseases. A murine model of $R$. parkeri infection using $\mathrm{C} 3 \mathrm{H} /$ $\mathrm{HeJ}$ mice showed that a portion of intradermally inoculated mice developed eschar-like necrotic lesions at the initial needle injection site that may be useful in understanding aspects of the pathogenesis and immunology of a nonfatal SFG rickettsiosis. ${ }^{10} \mathrm{C} 3 \mathrm{H} / \mathrm{HeN}$ mice inoculated intravenously with $R$. conorii develop disseminated endothelial infection, become ill on day 4, and die on day 5 or 6 as a result of vascular injury-based meningoencephalitis and interstitial pneumonia. ${ }^{11}$ The $\mathrm{C} 3 \mathrm{H} / \mathrm{HeN}$ mouse strain provides the best model to date for examining rickettsial disease with endothelial infection and injury. ${ }^{11}$ Rickettsia australis establishes a dose-dependent fatal infection model in C57BL/6 (B6) mice, ${ }^{12}$ and therefore also provides a very useful tool for mechanistic studies of both immunity to and pathogenesis of rickettsial diseases.

Because SFG rickettsiae are transmitted primarily via tick vectors, the role of tick saliva in the dissemination and transmission of rickettsial agents is of considerable interest. Intradermal inoculation of $R$. conorii into $\mathrm{C} 3 \mathrm{H} / \mathrm{HeJ}$ mice in the presence of saliva from Rhipicephalus sanguineus ticks inhibits the proinflammatory effects of IL- $1 \beta$ and NF- $\kappa B .{ }^{13}$ In addition, intradermal needle inoculation of $R$. parkeri in the presence of uninfected feeding ticks results in significantly increased rickettsial loads in the organs when compared with mice that are infected by intradermal needle inoculation of $R$. parkeri without tick feeding. ${ }^{14}$ Additional work has provided preliminary data toward the development of an immunocompetent rhesus macaque model using intradermal inoculation of $R$. parkeri during A. maculatum feeding with eschar formation and increased inflammatory markers in serum. ${ }^{15}$ Guinea pig animal models also have been used to determine a few aspects of transmission and disease presentation ${ }^{16,17}$; however, the guinea pig model has several significant limitations with respect to investigations of the immune response and disease pathways in the vertebrate host because of a paucity of reagents for this species, lack of gene knockout animals, and a paucity of inbred animals for adaptive transfer experiments. Although many aspects of rickettsial diseases have been studied using animal models, the complex interaction among the infected vector tick, the host, and the pathogen has not been addressed experimentally because of the absence of a tick transmission mouse model of SFG rickettsiosis that mimics naturally occurring infection that is essential for studying the natural events of immunity and pathogenesis of these tick-borne diseases.

\section{Materials and Methods}

\section{Experimental Design}

Our experiments were performed in two completely separate phases. Because tick transmission experiments are very elaborate to perform, the most susceptible mice strain to $R$. parkeri infection by needle inoculation first was determined. Thereafter, the selected strain was used to evaluate ticktransmitted infection using A. maculatum-carrying $R$. parkeri.

\section{Phase 1: Identification of a Suitable Mouse Strain for} $R$. parkeri Infection

\section{Bacteria/Inoculum}

$R$. parkeri (the maculatum 20 strain was originally gifted from Chip Pretzman, Ohio Department of Health, Columbus, OH) was maintained and amplified in cell culture using Vero cells. Bacterial inoculum was prepared by sonication of highly infected cell culture, for pathogen release, followed by ultracentrifugation to concentrate the bacteria, and further dilution to the desired inoculum dose. ${ }^{18}$ The concentration of viable rickettsiae was determined by plaque assay as described in previous studies. ${ }^{19}$ Species identity was confirmed by sequencing the sca5, htrA, and sca0 genes in the DNA extracted from the infected cell culture and inoculum samples, in triplicate, using a 3130xl Genetic Analyzer (Life Technologies, Carlsbad, CA). The inoculum dose used for intravenous infection was determined to be approximately $1.2 \times 10^{7}$ bacteria/mouse.

\section{Animals and Infection}

Six- to 8-week-old female C57BL/6, BALB/c, and C3H/HeN mice (Envigo, Inc., Houston, TX), in groups of five animals (two as negative controls) per time point, were inoculated by the i.v. route with $R$. parkeri or control medium to identify the mouse strain with the greatest susceptibility to infection to be used for the tick transmission studies.

\section{Collection of Samples}

Samples of spleen, liver, lung, brain, heart, thymus, kidney, lymph nodes (brachial and inguinal), and skin were collected from the needle-inoculated animals. Parts of all samples were fixed in $10 \%$ neutral buffered formalin for histopathology, and additional parts of spleen, liver, lung, brain, and skin were obtained for determination of the bacterial burdens. In addition, whole blood samples were obtained by cardiac puncture before euthanasia for separation of serum to be used for titration of antibodies against R. parkeri. For initial studies of the most appropriate mouse strain to be used further to develop the tick transmission models, samples were collected during the acute infection (day 6 after infection) and after recovery at day 30 after infection from intravenously infected $\mathrm{C} 57 \mathrm{BL} / 6, \mathrm{C} 3 \mathrm{H} / \mathrm{HeN}$, and $\mathrm{BALB} / \mathrm{c}$ mice.

\section{Determination of Bacterial Loads}

Samples of organs were processed for DNA extraction using the DNeasy Blood and Tissue Kit (Qiagen, Valencia, CA) with a few modifications. Detection and quantification of rickettsial DNA were performed by targeting the citrate synthase gene $(g l t A)$ by real-time PCR using an iCycler IQ 
from Bio-Rad Laboratories (Hercules, CA), as described previously. ${ }^{20,21}$ Briefly, the rickettsial burdens were determined using the following primers: gltA, 5'-GAGAGAAAATTATATCCAAATGTTGAT- ${ }^{\prime}$ (forward) and 5'-AGGGTCTTCGTGCATTTCTT-3' (reverse; Sigma-Genosys, St. Louis, MO); and gltA, 5'-CATTGTGCCATCCAGCCTACGGT-3' (Biosearch Technologies, Novato, CA). The results were normalized to the DNA concentration of the same sample and expressed as a copy number of gltA rickettsial genes per microgram of DNA. Tissues from uninfected mice served as negative controls.

\section{Histopathology}

After formalin fixation, paraffin embedding, and sectioning, tissue samples were stained with hematoxylin and eosin for microscopic evaluation and identification of pathologic changes.

\section{Determination of Antibody Titer by Immunofluorescence Assay}

The serum samples of mice infected by the i.v. route were assayed for $\operatorname{IgG}$ antibodies against $R$. parkeri antigen by immunofluorescence assay (IFA). Vero cells infected with $R$. parkeri and uninfected cell controls were coated on IFA slides and fixed in acetone. Serum samples were added to the slides in serial twofold dilutions. The titer of serum-specific antibody was evaluated by fluorescent microscopy, using positive (anti-spotted fever group rickettsia antibody) and negative (naive mouse serum) control samples and evaluation of rickettsial morphology in positive samples for specificity.

\section{Phase 2: Development of the Tick Transmission Model}

\section{Tick Vector/Bacteria}

For the vector tick transmission studies, field-collected A. maculatum ticks naturally carrying $R$. parkeri (most closely related to the Portsmouth strain) were generously gifted by Dr. Shahid Karim (University of Southern Mississippi, Hattiesburg, MS) ${ }^{22,23}$ The infected colony was established in 2014 (12\% to $32 \%$ carrying $R$. parkeri ${ }^{22,24}$ ) and has been maintained under laboratory conditions thereafter. The microbiome of the A. maculatum did not show other pathogens besides $R$. parkeri, as described by Budachetri et al. ${ }^{22}$ The analysis of sca0 gene sequences showed $100 \%$ homology of the maculatum 20 strain, Portsmouth strain, and R. parkeri in $A$. maculatum ticks. Five to nine nymphs of $A$. maculatum-carrying $R$. parkeri per mouse were used to study the infection transmitted by ticks. The same number of uninfected A. maculatum nymphs was used to infest control animals.

\section{Animals and Infection}

$R$. parkeri infection after i.v. inoculation (details in Clinical and Pathologic Effects of I.V. Infection by R. parkeri in Different Strains of Mice: Phase I) showed that $\mathrm{C} 3 \mathrm{H} / \mathrm{HeN}$ mice were the most suitable strain to use for the development of an $R$. parkeri vector tick transmission model of SFG rickettsiae because they developed the greatest bacterial loads. After determining the mouse strain with the highest levels of infection and greatest clinical signs, tick infestation was performed in groups of three mice, with $R$. parkericarrying A. maculatum nymphs. Samples were collected at three predetermined time points when the animals were euthanized to evaluate infection, disease development, and pathologic lesions in comparison with mice infested with uninfected ticks (groups of two animals per time point). A restrained feeding capsule was used to locate the area of sample collection at the site of tick attachment.

\section{Collection of Samples}

Tissue samples were collected as described in phase 1 . For the development of the A. maculatum tick transmission model of $R$. parkeri infection, the samples were obtained during the course of infection at 24 hours after tick attachment and days 6 and 12 after tick attachment to evaluate the disease progression.

\section{Bacterial Load, Histopathology, and Antibody Titer}

The samples of tick-infested animals were processed for bacterial levels, histologic changes, and evaluation of antibody titers as described for phase 1 .

\section{Immunohistochemistry}

Tissue sections were prepared for immunohistochemical detection of rickettsial antigen. Samples were first deparaffinized, hydrated, and treated with proteinase K (Dako, Carpinteria, CA) for antigen retrieval. Then the sections were incubated with polyclonal rabbit anti-spotted fever group rickettsial antibody at a dilution of 1:300 for 1 hour followed by biotinylated secondary goat anti-rabbit IgG antibody at 1:200 dilution (Vector Laboratories, Burlingame, CA), streptavidin-AP (Thermo Scientific, Waltham, MA), and Fast Red (Dako). The sections were counterstained with hematoxylin. Naive rabbit serum was as used as a negative primary antibody control.

\section{Ethics Statement}

All experiments were performed in accordance with the guidelines of the Office of Laboratory Animal Welfare and the American Veterinary Medical Association, with approval by the Institutional Animal Care and Use Committee of the University of Texas Medical Branch at Galveston, TX. A. maculatum ticks were maintained at the University of Southern Mississippi by a protocol approved by their Animal Care and Use Committee (Animal Welfare Assurance A3314-01).

\section{Results}

Clinical and Pathologic Effects of I.V. Infection by R. parkeri in Different Strains of Mice: Phase 1

R. parkeri induced subclinical infection even with a large infectious dose administered by the i.v. route in $\mathrm{C} 57 \mathrm{Bl} / 6$ 
mice, which did not present any clinical signs or behavioral changes during the course of infection. The evaluation of bacterial levels during infection showed low levels of copies of rickettsial gltA at day 6 after infection, as determined by real-time PCR. The lungs contained $R$. parkeri gltA DNA, but the bacterial burden varied in other organs (Figure 1A). Evaluation of the histopathologic changes in tissues at this time point showed extensive hepatic lesions, with areas of coagulative necrosis surrounded by infiltration of macrophages, lymphocytes, and polymorphonuclear neutrophils (PMNs) (Figure 2, A, B, D, and E, respectively). Several areas of inflammatory cellular infiltration consisting predominantly of perivascular lymphocytes and macrophages, but also distributed throughout the tissue, also were observed (Figure 2C). Numerous mitotic figures were present in all hepatic sections, suggesting a regenerative process. Infected C57BL/6 mice also had mild interstitial pneumonia, foci of peribronchovascular lymphocytic aggregates (Figure 2F), and focal lymphocytic meningitis.

Late in the infection (day 30 after infection), C57BL/6 mice showed prominent granulation tissue in liver tissue around the infarcts, indicating tissue repair and

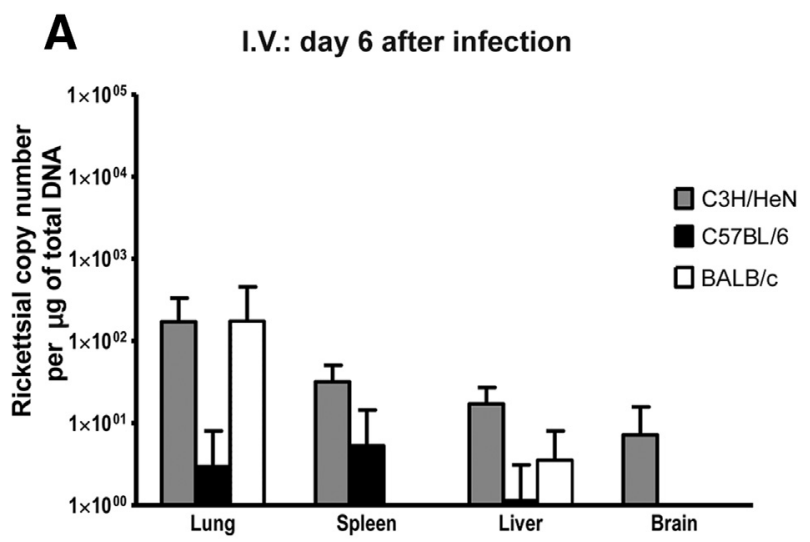

B Tick transmission: day 6 after tick attachment

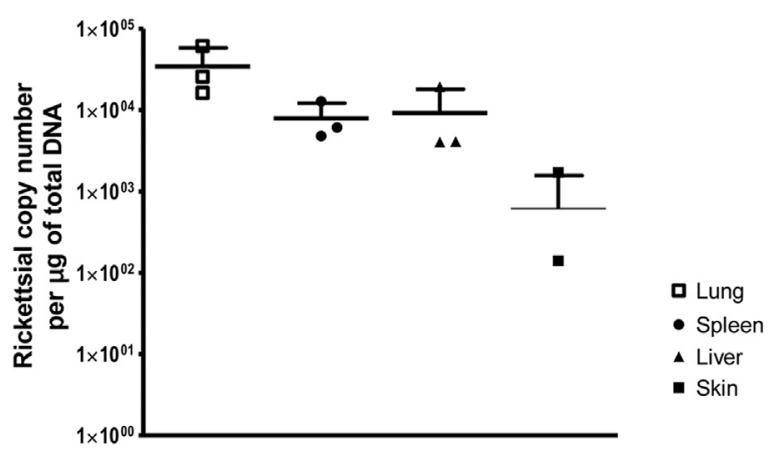

Figure 1 Bacterial loads in tissues during rickettsial infection after intravenous inoculations and by tick transmission. A: Levels of rickettsial citrate synthase gene copies in tissues at day 6 after intravenous infection by Rickettsia parkeri in different strains of mice. B: Levels of rickettsial citrate synthase gene copies in tissues from $\mathrm{C} 3 \mathrm{H} / \mathrm{HeN}$ mice infected with $R$. parkeri by tick transmission on day 6 after tick attachment. accumulation of lymphocytes around lung vessels and pulmonary bronchioles (Figure 2, G-I). The antirickettsial antibody response of infected animals at this time point indicated a strong induction of a host humoral immune response after i.v. rickettsial inoculation, with IFA titers of 1024 or higher.

BALB/c mice infected by i.v. injection of $R$. parkeri showed only a mild decrease in physical activity, but no other clinical manifestations. Similar to C57BL/6 mice, they contained low levels of rickettsial gltA copies at day 6 after infection in the lungs, but with varied bacterial loads in other organs (Figure 1A). In addition, the histopathologic changes induced by $R$. parkeri infection in organs were similar to those observed in C57BL/6 mice, but less severe. $\mathrm{BALB} / \mathrm{c}$ mice showed lower IgG titers than C57BL/6 mice, with levels of approximately 512 .

In contrast, $\mathrm{C} 3 \mathrm{H} / \mathrm{HeN}$ mice developed lethargy, a mild hunched posture, ruffled fur, and decreased activity, with an onset at approximately days 6 to 7 after i.v. infection. These clinical manifestations persisted for 2 to 3 days until the animals recovered by day 9 or 10 after infection. These mice had bacterial DNA in all tissues evaluated at the time of clinical manifestations of infection, with the highest levels in the lungs. The peak of the rickettsial burden in all organs tested was on day 6 after infection. Although the clinical signs of infection were more prominent than in the other mouse strains, the infarcts in liver were less severe and cellular infiltration in lungs more diffuse, consistent with a reduced immune ability to control the rickettsial infection (Figure 3, A-E). An accumulation of lymphocytes also was observed surrounding the pulmonary blood vessels and airways (Figure 3F); however, they were present less frequently in this mouse strain than in C57BL/6 mice. The IgG IFA titers were lower than in the other strains, with levels of approximately 256 .

Evaluation of the results of the infection in mice inoculated intravenously with $R$. parkeri showed that the $\mathrm{C} 3 \mathrm{H} /$ $\mathrm{HeN}$ mouse strain had the most severe clinical manifestations, highest bacterial loads, and observed clinical disease course of the three mouse strains, with the best potential to be evaluated for the development of a successful tick transmission model.

\section{Murine Infection and Clinical-Pathologic Manifestations of $A$. maculatum Tick Transmission of R. parkeri: Phase 2}

$\mathrm{C} 3 \mathrm{H} / \mathrm{HeN}$ mice were infested with the nymphal stage of $A$. maculatum carrying $R$. parkeri. Clinical evaluation of animals infected by tick transmission showed the development of illness between days 8 and 10 after tick attachment, which represents a delay of approximately 2 days of the clinical manifestations compared with mice infected with the same rickettsial pathogen by the i.v. route. All animals showed a decrease in physical activity, became less responsive to environmental stimuli, and progressed to more severe clinical 

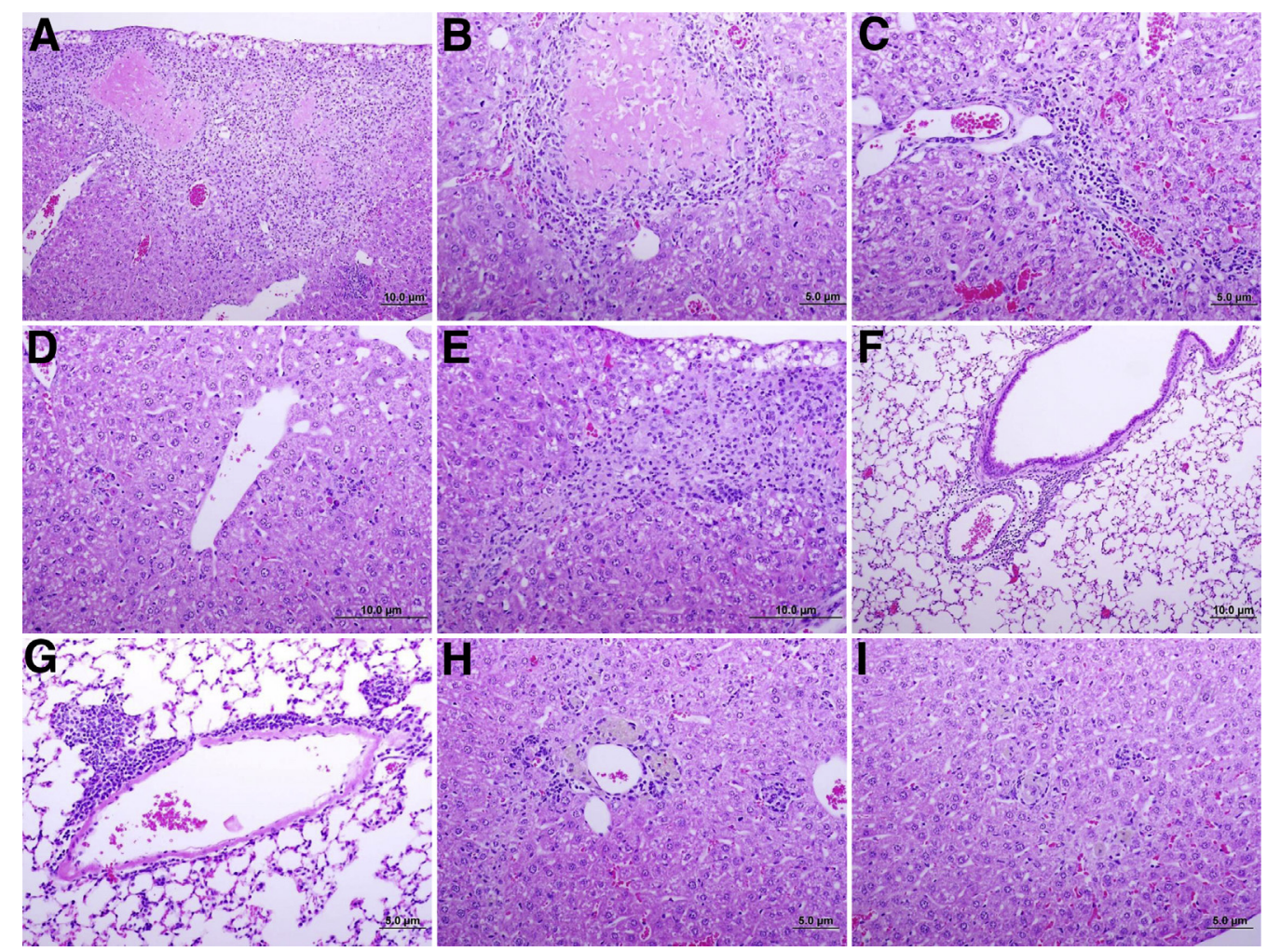

Figure 2 Histopathology of tissues from C57BL/6 mice infected with Rickettsia parkeri by i.v. inoculation. A and B: Extensive mononuclear inflammatory cells around regions of hepatic coagulative necrosis. C: Lymphocytic infiltration in a portal triad. D: Scattered small lobular foci of inflammatory cell accumulations in hepatic lobules. E: Hepatic massive inflammatory infiltration surrounds areas of necrosis and perivascular locations at day 6 after infection, in mice infected with $R$. parkeri by i.v. inoculation. F: Perivascular-peribronchial inflammatory infiltration in lung of intravenously infected mice 6 days after infection. G: Foci of lymphocytic infiltration in perivascular area in lungs of intravenously infected mice 30 days after infection. $\mathbf{H}$ and I: Multiple lobular foci of mononuclear cell accumulations in the liver of convalescent mice 30 days after infection.

manifestations, including ruffled fur and hunched posture. Rickettsial DNA was detected in the skin site of tick transmission at 24 to 36 hours after tick attachment, and also two of three animals already showed early bacterial dissemination in tissues such as lung and spleen at this time. During the peak of clinical illness, the quantity of bacteria in all tissues tested (spleen, liver, lung, and skin) also reached the highest levels, including the tick bite site in the skin (Figure 1B). At day 12
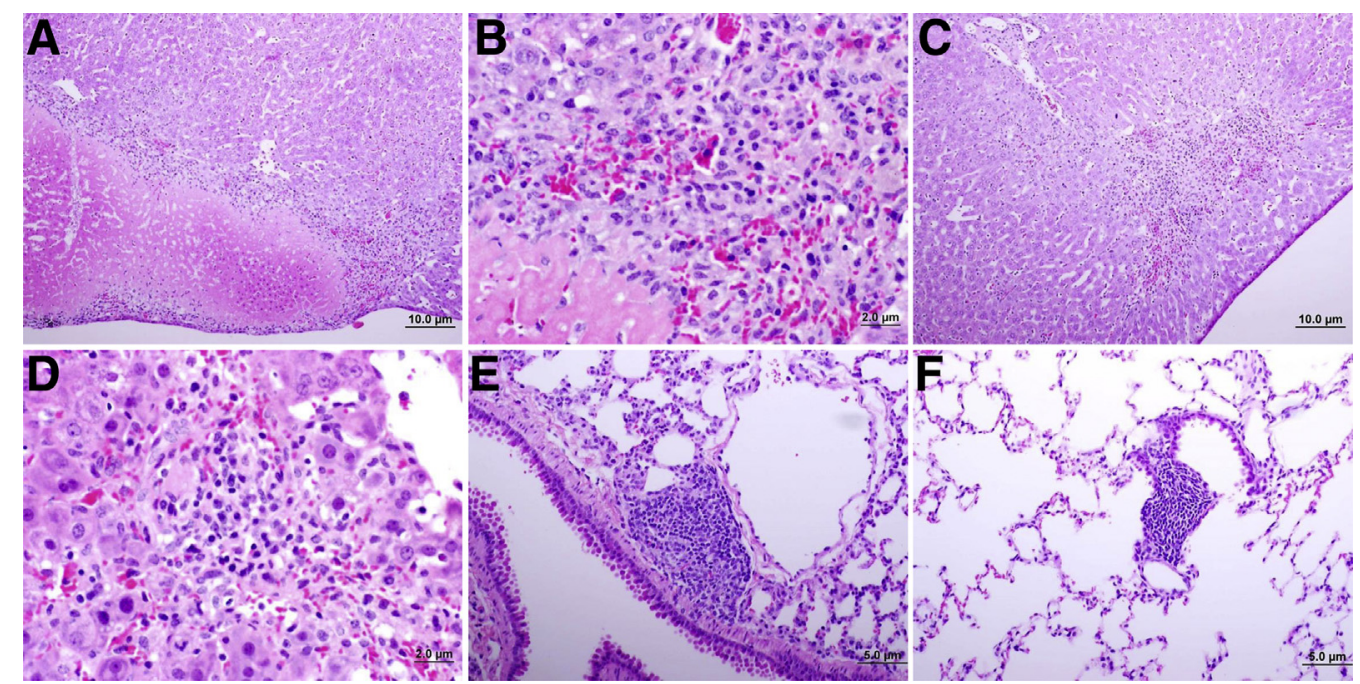

Figure 3 Histopathology of tissues from $\mathrm{C} 3 \mathrm{H} / \mathrm{HeN}$ mice infected intravenously with Rickettsia parkeri. A and B: Areas of infarction surrounded by granulation tissue and inflammatory cells in liver 6 days after infection. $\mathbf{C}$ and D: Inflammatory infiltration in hepatic tissue of animals 6 days after infection. E: Focus of lymphocytic inflammation in lung 6 days after infection. F: Persistence of inflammatory infiltration in lung 30 days after infection. 

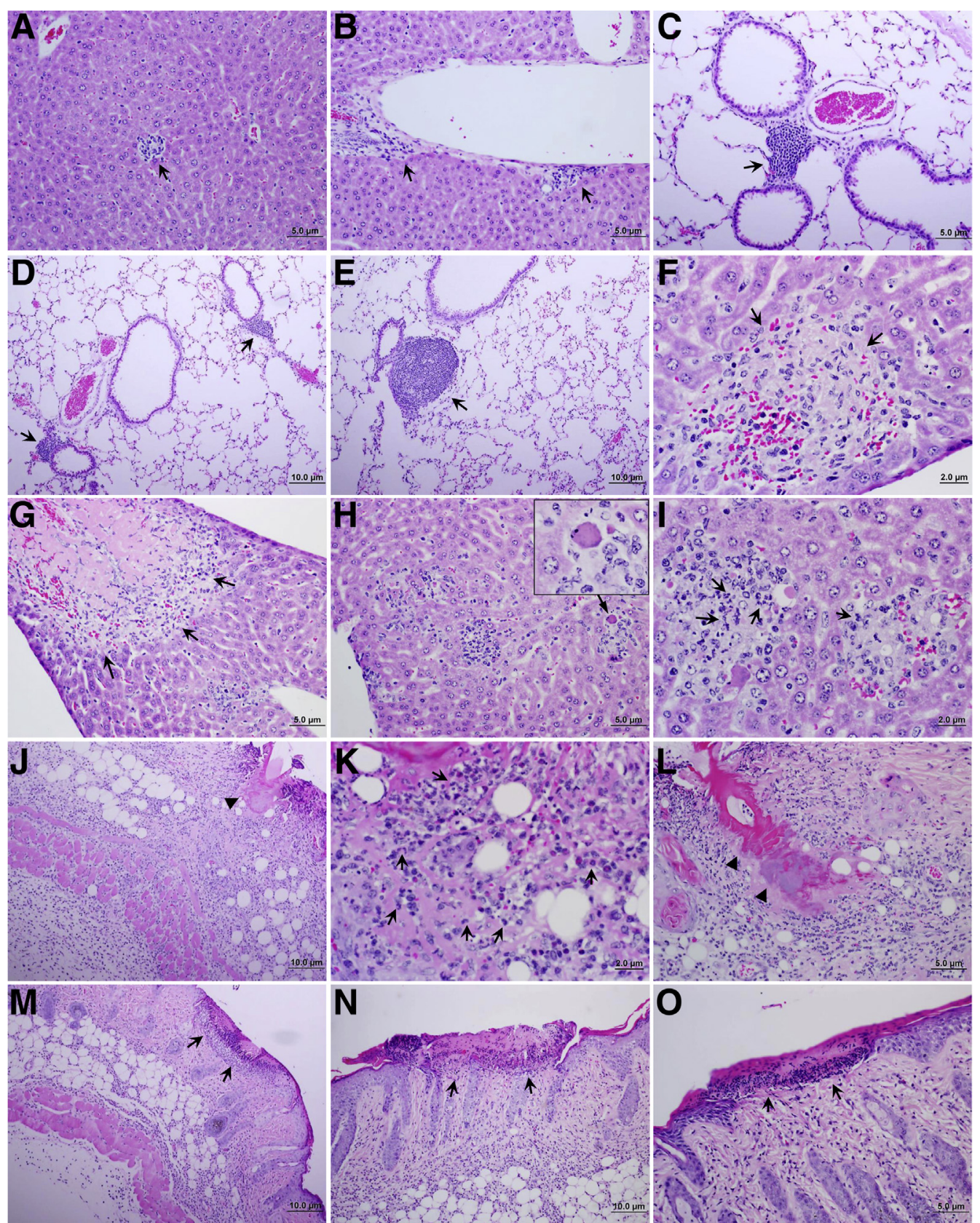

Figure 4 Histopathologic lesions in tissues of mice infected with Rickettsia parkeri by tick transmission. A and B: Foci of inflammatory cells and perivascular infiltration in liver 24 hours after tick attachment (arrows). C and D: Foci of lymphocytic infiltration in peribronchial-perivascular areas of the lung of mice infected by tick transmission observed 24 hours after tick attachment (arrows). E: Similar lesions in lung of mice 6 days after tick attachment (arrow). $\mathbf{F}$ and G: Areas of infarction (arrows). H and I: Inflammatory infiltration with apoptotic cells (H; arrow; inset shows higher magnification of the apoptotic cell) and polymorphonuclear neutrophils (PMNs; I; arrows) in liver of mice 6 days after infection with $R$. parkeri by tick transmission. $\mathbf{J}-\mathbf{L}$ : Remarkable presence of PMNs (arrows) and other inflammatory cells at the $R$. parkeri-carrying Amblyomma maculatum tick feeding site in the skin (arrowheads show tick mouth parts and cement insertion) 6 days after tick attachment. $\mathbf{M}-\mathbf{0}$ : Area of necrosis of dermis and epidermis at skin site of $R$. parkeri-carrying tick attachment and feeding at day 12 after tick attachment (arrows).

after tick attachment only two mice still maintained rickettsial DNA detected by real-time PCR, one in liver and the other in the skin site of tick feeding. The skin was the only tissue that contained rickettsial DNA in at least one animal at all time points evaluated throughout the course of the experimental infection. The $R$. parkeri loads in the tissues of animals infected by tick transmission were approximately $10^{4}$ copies/ $\mu \mathrm{g}$ of total DNA (Figure 1). Initial foci of inflammatory infiltration in liver tissue were observed as early as 24 to 36 hours after $R$. parkeri-carrying tick attachment, with frequent large foci containing apoptotic cells surrounded by lymphocytes, macrophages, and PMNs (Figure 4, A and B). Hepatic lesions at day 6 after tick attachment consisted of the presence of inflammatory foci with the distinct presence of 


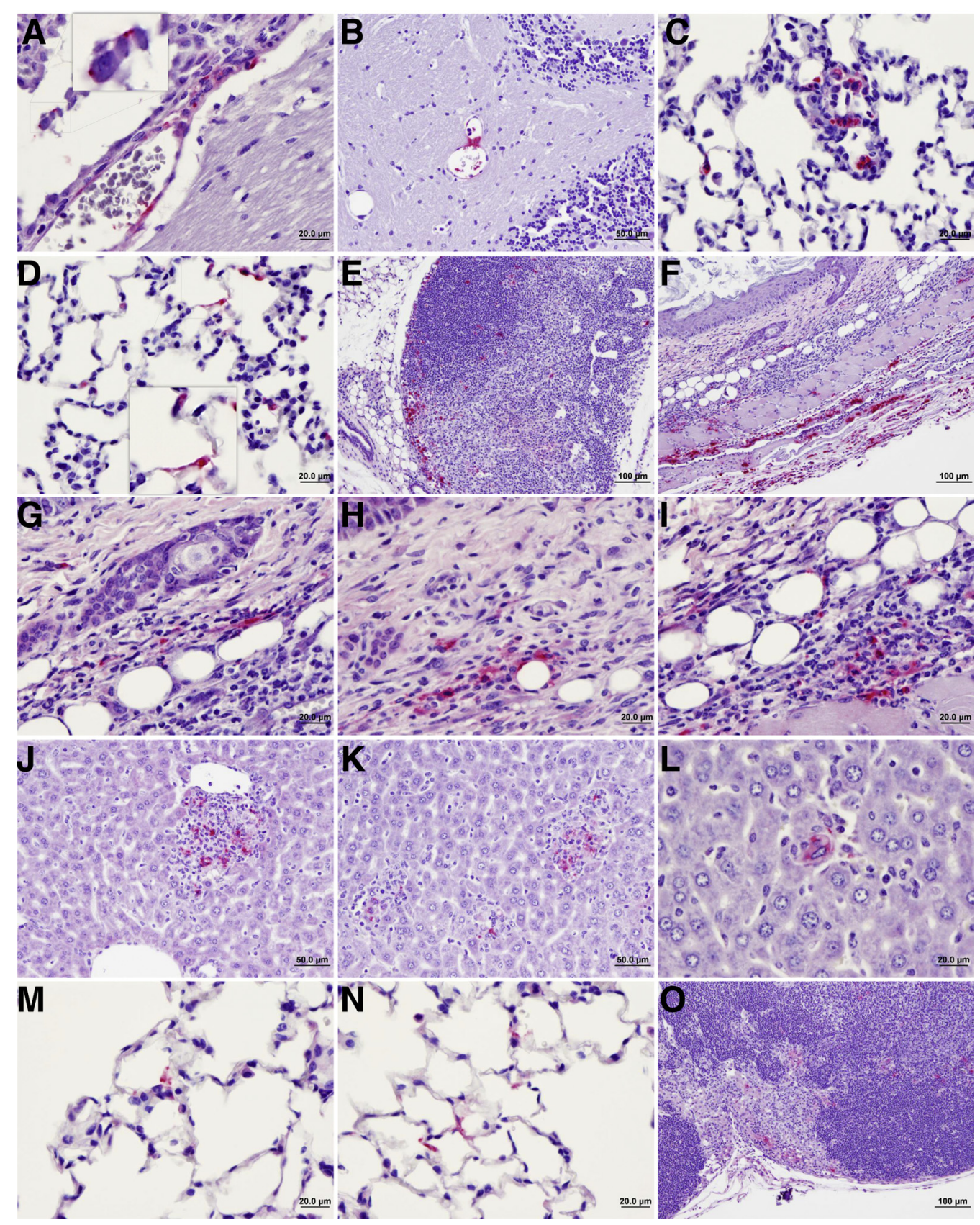

Figure 5 Immunohistochemistry of Rickettsia parkeri antigen in tissues from intravenously infected $\mathrm{C} 3 \mathrm{H} / \mathrm{HeN}$ mice and by tick transmission. A and B: Presence of rickettsiae in brain of intravenously infected mice 6 days after infection, showing endothelial and perivascular rickettsial antigen. Inset shows individual rickettsial antigen. C and D: Identification of rickettsial infection in lung of intravenously infected mice. Inset shows individual rickettsial antigen. E: Axillary lymph node infected with rickettsiae in intravenously inoculated mice 6 days after infection. F-I: Skin area of tick transmission with a massive presence of rickettsial antigen in many cell types 6 days after tick attachment, including perivascular infection. J-L: Rickettsial antigen present within areas of inflammatory infiltration in liver during tick transmission (day 6 after tick attachment). M and $\mathbf{N}$ : Infection of lungs with visualization of individual bacteria in the lungs of $R$. parkeri infection by tick transmission 6 days after tick attachment. 0: Draining axillary lymph node related to the site of tick attachment on day 6 after tick attachment, showing the presence of rickettsial antigen.

PMNs (Figure 4, $\mathrm{H}$ and I). Liver also contained areas of coagulative necrosis that were surrounded by inflammatory cells, mainly macrophages (Figure 4, F and G). The regenerative process was characterized in liver by the presence of an increased number of hepatocytic mitotic figures throughout the tissue. Interestingly, at day 12 after tick attachment, fewer inflammatory foci were identified in liver, and fewer PMNs were observed compared with early infection. Peribronchial and perivascular infiltrations of lymphocytes and macrophages also were observed in the lungs of infected animals at 24 to 36 hours after tick attachment (Figure 4, C and D); larger foci were present at day 6 after tick attachment (Figure 4E), and small infiltrates were observed at 12 days after tick attachment. Skin at the tick feeding site 
showed inflammatory infiltration of mononuclear leukocytes and numerous PMNs. Some of the tick feeding areas contained foci of necrotic epidermis and dermis typical of eschars, surrounded by inflammatory cellular infiltration observed in infected mice at day 12 after tick attachment (Figure 4, J-O).

Immunostaining showed the presence of rickettsial antigen in some clearly identified endothelial cells in brain, liver, and endocardium. Other cells also contained rickettsial antigen during infection by tick transmission, potentially Kupffer cells, and splenic macrophages. Interestingly, areas of massive presence of rickettsial antigen were observed in all the skin layers at 6 days after tick attachment, within several cell types. Some areas of inflammatory infiltration, especially in liver, surrounded rickettsiae-infected cells (Figure 5).

Interestingly, mice infected with $R$. parkeri by A. maculatum tick transmission produced an IFA IgG antibody response as early as day 12 after tick attachment at a titer of 512 or higher, suggesting a strong humoral response.

\section{Discussion}

Our experiments showed a murine model of tick transmission of $R$. parkeri, an emerging SFG rickettsial pathogen with a broad distribution in the Western hemisphere. With this model we will be able to study the complex tick-host-pathogen interface, and the effects on the host immune response and consequent disease development. Recently, reports of $R$. parkeri infection were published showing an increase in human cases of disease. ${ }^{3-5,25-28}$ However, little is known about the disease establishment or pathogenesis. Patients with $R$. parkeri disease remain moderately ill for 7 to 10 days with fever, vesicular or pustular rash (in approximately $40 \%$ of patients), and occurrence of eschar (in approximately $90 \%$ of the patients). ${ }^{25}$ Previous experiments have addressed developing animal models to evaluate $R$. parkeri infection ${ }^{10,14,15,17}$; however, the closest models to the natural transmission of infection used needle inoculation of bacteria at the site of uninfected tick feeding. These studies have focused on the generation of skin lesions by $R$. parkeri and the role of the tick feeding on the development of an eschar. ${ }^{14,15}$ Interestingly, results obtained from these models showed increased PMNs in the skin site of infection when needle inoculation of rickettsiae was combined with tick feeding. The importance of tick feeding in enhancing infection by rickettsial pathogens also was shown in other studies, showing $R$. parkeri interference with tick gene expression and suggesting the potential importance of the bacterial origin from the vector for enhancement of transmission. ${ }^{22,29,30}$ Taken together these research findings emphasize the importance of a natural model of infection with bacterial passage through the tick vector before transmission to the skin of the vertebrate host by tick feeding.
Our findings showed high bacterial loads after tick transmission shown by real-time quantitative PCR and immunostaining systemically, but even more intense in the skin site of tick feeding. Although endothelial cells were well characterized as the principal target cell during systemic infection, as other researchers have shown, at the dermal area of transmission many cell types contained rickettsiae. This finding emphasizes the value of sampling the skin inoculation site for the diagnosis of disease during the acute infection, as observed in studies of eschar swabs from human patients with $R$. parkeri and other SFG rickettsial infections. The skin site of experimental tick transmission showed the development of an eschar-like lesion, with necrotic dermis and epidermis and infiltration of inflammatory cells into the area of tick feeding, similar to the described pathologic changes in human cases of $R$. parkeri infection. ${ }^{31}$ The infection also induced inflammatory infiltration in other infected organs during the course of illness. Interestingly, the prominent presence of PMNs and rickettsial antigen was observed in skin and some of the affected organs, as occurs in human cases. ${ }^{25}$ The continuous bacterial inoculation during the period of tick feeding would be technically challenging to reproduce by needle inoculation because feeding takes many days. Increased infection levels in this model might have resulted from a higher inoculum by the arthropod, but it also may have resulted from a tick feeding effect on the host immune response favoring infection by the rickettsial pathogen. These results also show early induction of humoral immunity occurring by 12 days after tick attachment.

This study showed the development of a murine model of A. maculatum tick vector transmission of SFG rickettsiae involving all aspects of the natural infection. The complexity associated with this natural model of tick transmission comprises the following: i) naturally acquired rickettsial pathogen by the tick vector (transovarially maintained colony of infected tick from nature); ii) horizontal transmission with bacterial dissemination within the tick and maintenance through the tick life stages (during colony maintenance); and iii) transmission of the pathogen to the vertebrate host during prolonged tick feeding (presented in this article). This model will be useful in evaluating the mechanisms of rickettsial manipulation of the vector tick gene expression, rickettsial genes expressed in the tick salivary glands and skin feeding site, and the effects of tick saliva on the vertebrate host to induce infection and disease.

\section{Acknowledgments}

We thank the Galveston National Laboratory-Animal Resource Center personnel, for research support during the performance of this project. A. maculatum ticks naturally carrying $R$. parkeri were a gift from Dr. Shahid Karim (University of Southern Mississippi, Hattiesburg, MS). 


\section{References}

1. Wright CL, Sonenshine DE, Gaff HD, Hynes WL: Rickettsia parkeri transmission to Amblyomma americanum by cofeeding with Amblyomma maculatum (acari: ixodidae) and potential for spillover. J Med Entomol 2015, 52:1090-1095

2. Herrick KL, Pena SA, Yaglom HD, Layton BJ, Moors A, Loftis AD, Condit ME, Singleton J, Kato CY, Denison AM, Ng D, Mertins JW, Paddock CD: Rickettsia parkeri rickettsiosis, Arizona, USA. Emerg Infect Dis 2016, 22:780-785

3. Biggs HM, Behravesh CB, Bradley KK, Dahlgren FS, Drexler NA, Dumler JS, Folk SM, Kato CY, Lash RR, Levin ML, Massung RF, Nadelman RB, Nicholson WL, Paddock CD, Pritt BS, Traeger MS: Diagnosis and management of tickborne rickettsial diseases: Rocky Mountain Spotted Fever and other spotted fever group rickettsioses, ehrlichioses, and anaplasmosis-United States. MMWR Recomm Rep 2016, 65:1-44

4. Paddock CD, Goddard J: The evolving medical and veterinary importance of the gulf coast tick (acari: ixodidae). J Med Entomol 2015, 52:230-252

5. Drexler NA, Dahlgren FS, Heitman KN, Massung RF, Paddock CD, Behravesh CB: National surveillance of spotted fever group rickettsioses in the United States, 2008-2012. Am J Trop Med Hyg 2016, 94:26-34

6. Nava S, Elshenawy Y, Eremeeva ME, Sumner JW, Mastropaolo M, Paddock CD: Rickettsia parkeri in Argentina. Emerg Infect Dis 2008, 14:1894-1897

7. Weck B, Dall'Agnol B, Souza U, Webster A, Stenzel B, Klafke G, Martins JR, Reck J: Spotted fever group rickettsia in the pampa biome, Brazil, 2015-2016. Emerg Infect Dis 2016, 22: 2014-2016

8. Weck B, Dall'Agnol B, Souza U, Webster A, Stenzel B, Klafke G, Martins JR, Reck J: Rickettsia parkeri in Amblyomma dubitatum ticks in a spotted fever focus from the Brazilian Pampa. Acta Trop 2017, 171:182-185

9. Venzal JM, Portillo A, Estrada-Peña A, Castro O, Cabrera PA, Oteo JA: Rickettsia parkeri in Amblyomma triste from Uruguay. Emerg Infect Dis 2004, 10:1493-1495

10. Grasperge BJ, Reif KE, Morgan TD, Sunyakumthorn P, Bynog J, Paddock CD, Macaluso KR: Susceptibility of inbred mice to Rickettsia parkeri. Infect Immun 2012, 80:1846-1852

11. Walker DH, Popov VL, Wen J, Feng HM: Rickettsia conorii infection of $\mathrm{C} 3 \mathrm{H} / \mathrm{HeN}$ mice. A model of endothelial-target rickettsiosis. Lab Invest 1994, 70:358-368

12. Walker DH, Olano JP, Feng HM: Critical role of cytotoxic T lymphocytes in immune clearance of rickettsial infection. Infect Immun 2001, 69:1841-1846

13. Milhano N, Saito TB, Bechelli J, Fang R, Vilhena M, DE Sousa R, Walker DH: The role of Rhipicephalus sanguineus sensu lato saliva in the dissemination of Rickettsia conorii in $\mathrm{C} 3 \mathrm{H} / \mathrm{HeJ}$ mice. Med Vet Entomol 2015, 29:225-229

14. Grasperge BJ, Morgan TW, Paddock CD, Peterson KE, Macaluso KR: Feeding by Amblyomma maculatum (acari: ixodidae) enhances Rickettsia parkeri (rickettsiales: rickettsiaceae) infection in the skin. J Med Entomol 2014, 51:855-863

15. Banajee KH, Embers ME, Langohr IM, Doyle LA, Hasenkampf NR, Macaluso KR: Amblyomma maculatum feeding augments Rickettsia parkeri infection in a rhesus macaque model: a pilot study. PLoS One 2015, 10:e0135175
16. Levin ML, Snellgrove AN, Zemtsova GE: Comparative value of blood and skin samples for diagnosis of spotted fever group rickettsial infection in model animals. Ticks Tick Borne Dis 2016, 7:1029-1034

17. Wright CL, Gaff HD, Sonenshine DE, Hynes WL: Experimental vertical transmission of Rickettsia parkeri in the Gulf Coast tick, Amblyomma maculatum. Ticks Tick Borne Dis 2015, 6:568-573

18. Ammerman NC, Beier-Sexton M, Azad AF: Laboratory maintenance of Rickettsia rickettsii. Curr Protoc Microbiol 2008, Chapter 3:3A.5

19. Fang R, Ismail N, Shelite T, Walker DH: CD4+ CD25+ Foxp3- Tregulatory cells produce both gamma interferon and interleukin-10 during acute severe murine spotted fever rickettsiosis. Infect Immun 2009, 77:3838-3849

20. Xin D, El Karkouri K, Robert C, Raoult D, Fournier PE: Genomic comparison of Rickettsia honei strain RBT and other Rickettsia species. J Bacteriol 2012, 194:4145

21. Fang R, Ismail N, Walker DH: Contribution of NK cells to the innate phase of host protection against an intracellular bacterium targeting systemic endothelium. Am J Pathol 2012, 181:185-195

22. Budachetri K, Browning RE, Adamson SW, Dowd SE, Chao CC, Ching WM, Karim S: An insight into the microbiome of the Amblyomma maculatum (acari: ixodidae). J Med Entomol 2014, 51:119-129

23. Budachetri K, Kumar D, Karim S: Catalase is a determinant of the colonization and transovarial transmission of Rickettsia parkeri in the Gulf Coast tick Amblyomma maculatum. Insect Mol Biol 2017, 26 : 414-419

24. Budachetri K, Kumar D, Crispell G, Beck C, Dasch G, Karim S: The tick endosymbiont Candidatus Midichloria mitochondrii and selenoproteins are essential for the growth of Rickettsia parkeri in the Gulf Coast tick vector. Microbiome 2018, 6:141

25. Paddock CD, Finley RW, Wright CS, Robinson HN, Schrodt BJ, Lane CC, Ekenna O, Blass MA, Tamminga CL, Ohl CA, McLellan SL, Goddard J, Holman RC, Openshaw JJ, Sumner JW, Zaki SR, Eremeeva ME: Rickettsia parkeri rickettsiosis and its clinical distinction from Rocky Mountain spotted fever. Clin Infect Dis 2008, $47: 1188-1196$

26. Romer Y, Nava S, Govedic F, Cicuttin G, Denison AM, Singleton J, Kelly AJ, Kato CY, Paddock CD: Rickettsia parkeri rickettsiosis in different ecological regions of Argentina and its association with Amblyomma tigrinum as a potential vector. Am J Trop Med Hyg 2014, 91:1156-1160

27. Lado P, Costa FB, Verdes JM, Labruna MB, Venzal JM: Seroepidemiological survey of Rickettsia spp. in dogs from the endemic area of Rickettsia parkeri rickettsiosis in Uruguay. Acta Trop 2015, 146:7-10

28. Melo AL, Alves AS, Nieri-Bastos FA, Martins TF, Witter R, Pacheco TA, Soares HS, Marcili A, Chitarra CS, Dutra V, Nakazato L, Pacheco RC, Labruna MB, Aguiar DM: Rickettsia parkeri infecting free-living Amblyomma triste ticks in the Brazilian Pantanal. Ticks Tick Borne Dis 2015, 6:237-241

29. Adamson SW, Browning RE, Budachetri K, Ribeiro JM, Karim S: Knockdown of selenocysteine-specific elongation factor in Amblyomma maculatum alters the pathogen burden of Rickettsia parkeri with epigenetic control by the $\operatorname{Sin} 3$ histone deacetylase corepressor complex. PLoS One 2013, 8:e82012

30. Crispell G, Budachetri K, Karim S: Rickettsia parkeri colonization in Amblyomma maculatum: the role of superoxide dismutases. Parasit Vectors 2016, 9:291

31. Myers T, Lalani T, Dent M, Jiang J, Daly PL, Maguire JD, Richards AL: Detecting Rickettsia parkeri infection from eschar swab specimens. Emerg Infect Dis 2013, 19:778-780 